\title{
The sparing phenomenon of cutaneous amyloidosis
}

\author{
Hui-Wen Zhou ${ }^{1,2}$, Cheng Tan ${ }^{1}$ \\ ${ }^{1}$ Department of Dermatology, Affiliated Hospital of Nanjing University of Chinese Medicine, Nanjing, China \\ ${ }^{2}$ Department of Dermatology, ShenZhen Traditional Chinese Medicine, ShenZhen, China
}

Adv Dermatol Allergol 2021; XXXVIII (4): 694-695

DOI: https://doi.org/10.5114/ada.2021.108928

The sparing phenomenon (SP) denotes a particular skin disease which spares an anatomic site or an area affected by other skin disorders [1]. SP occurs in miscellaneous skin conditions such as herpes zoster, drug eruptions, alopecia areata and so on. For the first time, both the nipple-areola complex and the depigmented skin in vitiligo were found to be spared in cutaneous amyloidosis.

Case 1 was a 37-year-old male who was referred with a 7-year history of lichenoid papules on the back and breast. There was mild itching. On examination, we observed extensive brownish macules, hyperkeratotic papules, and excoriation on the back. On the breast, these lesions coalesced to form a demarcated targetoid configuration. Strikingly, the nipple and inner part of areola were spared (Figure 1). Skin biopsy of lesions on the breast showed hyperkeratosis, acanthosis, and papillomatosis. The dermis was showing deposits of amyloid confirmed by Congo red stain. The diagnosis of lichen amyloidosis sparing the nipple and areola was established. Case 2 was a 24-year-old woman with pathologically-proven vitiligo on the back for 7 years. Two years before this presentation, she developed brownish pruritic patches on the back next to the previous depigmented skin. On physical examination, the patient had homogeneous hypopigmented patches on the upper back. On the borders, there is a distinctive cut-off sign with outward brownish macules or keratotic papules which spares the achromic skin (Figure 2). The skin biopsy of the brown macular lesion was consistent with the diagnosis of cutaneous macule amyloidosis with the sign of amyloid deposition. The lesion was unresponsive to pimecrolimus 1\% cream for 3 months. Only itch was attenuated.

Locus minoris resistentiae ( $\mathrm{Imr}$ ) denotes a site of the body that is the privileged localization of certain disorder as compared to the remaining part [2]. Köbner phenomenon, isotopic response, and Renbök phenomenon all are good examples of Imr. In the presence of locus maioris resistentiae (LMR), skin diseases (occasionally developed upon ionizing radiation or localized trauma) can selectively spare a mosaic area, another unrelated pre-existing skin disorder (reverse Köbner's isomorphic response or reverse Renbök phenomenon), postherpetic site (Wolf's post-herpetic isotopic nonresponse), a burn scar (isocaumatopic nonresponse) or the anatomic site of the nipple and areola (Bork-Baykal phenomenon) [3].

The sparing of an anatomic site occurs in a diversity of skin disorders $[1,4]$. There is an anecdotal report that atopic dermatitis usually spares the napkin area. X-linked recessive ichthyosis spares the rhomboidal region of body folds. Tinea cruris amnesties the skin of the scrotum. In Bork-Baykal phenomenon, congenital melanocytic nevus surrounds the nipple and areola but spares the complex [4]. We meticulously noted that the presence of "nipple sparing" in cutaneous macular amyloidosis of the breast. Cutaneous lesion of our patient coalesced but spared both the nipple and inner part of the areola, leaving a demarcated targetoid configuration. Such islands of uninvolved areas are unusual for cutaneous amyloidosis since it generally causes diffused skin involvement. Cutaneous amyloidosis in our cases spared pre-existing vitiligo or the nipple and areola complex, which typically represented examples of LMR.

Although the precise cause remains unknown, the concept of "immunocompromised cutaneous district (ICD)' has been proposed for the pathogenesis of Imr and LMR [3]. An ICD is the vulnerability of a skin area than the remaining part, which facilitates (or impedes) the local onset of immunity-related skin disorders. Many contributing factors have been revealed including infections, mosaicism, chronic lymph stasis, burns, ultraviolet, traumas, neurologic disorders, intradermal vaccinations or a specific anatomic site $[3,5]$. We presume that skin in the nipple and areola complex has a certain anatomic predisposition to harbour amyloidosis. In the other aspect, the antecedent vitiligo may significantly impair

Address for correspondence: Cheng Tan MD, Department of Dermatology, Affiliated Hospital of Nanjing University of Chinese Medicine, 155 Hanzhong Road, 210029 Nanjing, China, phone: +86 2586617141, e-mail: tancheng@yeah.net Received: 7.08.2019, accepted: 27.10.2019. 

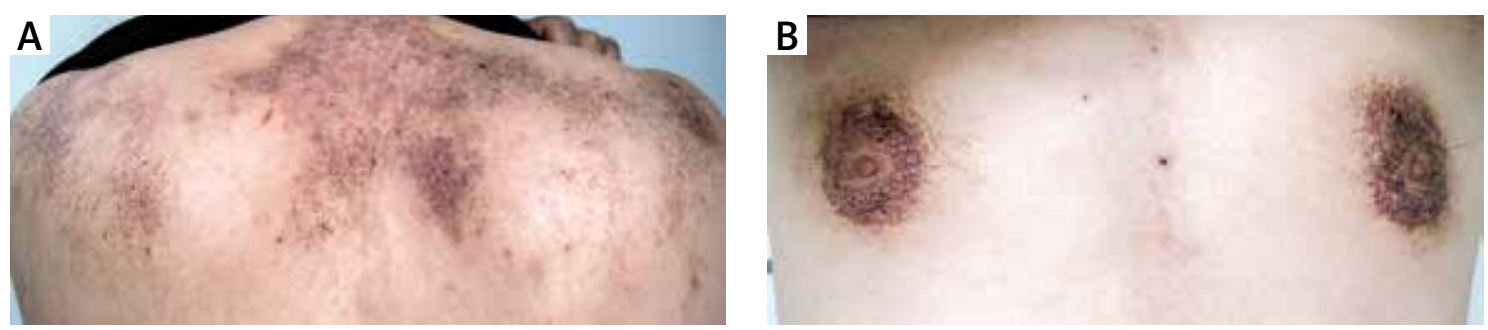

Figure 1. Clinical picture for case 1. Extensive brownish macules, hyperkeratotic papules, and excoriation on the back. On the breast, hyperkeratotic papules coalesced to form a demarcated targetoid configuration. The nipple and inner part of areola were spared

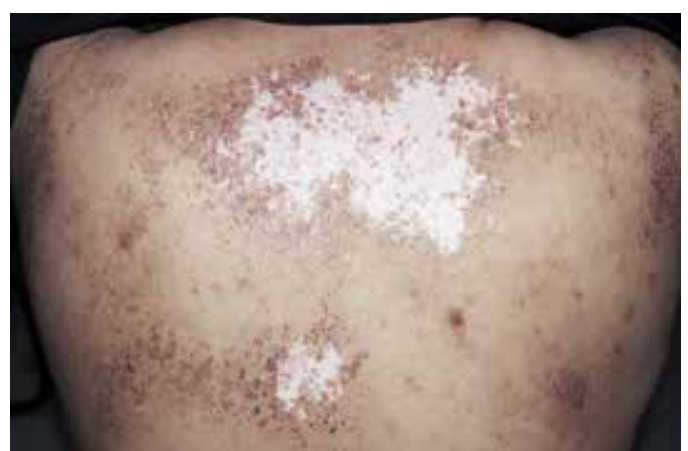

Figure 2. Clinical picture for case 2. Homogeneous hypopigmented patches on the upper back. On the borders, there is a distinctive cut-off sign with outward brownish macules or keratotic papules that remits the achromic skin

a local neuroimmune response, thus paving the way for the subsequent development of heterogeneous sparing phenomena in amyloidosis. Further investigation is needed to illuminate the exact factors precipitating the development of the sparing phenomenon in cutaneous amyloidosis.

We report 2 cases to increase the awareness of the sparing phenomenon of amyloidosis. Hopefully, this new observation further broadens the spectrum of the sparing phenomenon in dermatology.

\section{Acknowledgments}

The study was financially supported in part by the National Natural Science Foundation of China (Grant No. 81173400).

\section{Conflict of interest}

The authors declare no conflict of interest.

\section{References}

1. Pakran J. Sparing phenomena in dermatology. Indian J Dermatol Venereol Leprol 2013; 79: 545-50.

2. Lo Schiavo A, Ruocco E, Russo T, et al. Locus minoris resistentiae: an old but still valid way of thinking in medicine. Clin Dermatol 2014; 32: 553-6.

3. Ruocco V, Ruocco E, Piccolo V, et al. The immunocompromised district in dermatology: a unifying pathogenic view of the regional immune dysregulation. Clin Dermatol 2014; 32: $569-76$.

4. Happle R. The Bork-Baykal phenomenon: a revised eponymic designation for the sparing of nipple and areola in large melanocytic nevi involving the breast. J Eur Acad Dermatol Venereol 2017; 31: e214.

5. Baroni A, Buommino E, Piccolo V, et al. Alterations of skin innate immunity in lymphedematous limbs: correlations with opportunistic diseases. Clin Dermatol 2014; 32: 592-8. 